\title{
An Extension of the Transverse Wave Formulation to Model Stochastic Electromagnetic Fields
}

\author{
Johannes A. Russer*, Michael Haider*, Damienne Bajon ${ }^{\dagger}$, Sidina Wane ${ }^{\ddagger}$, and Peter Russer* \\ ${ }^{*}$ Institute for Nanoelectronics, Technische Universität München, Germany, e-mail: jrusser@tum. de, \\ $\dagger$ SUPAERO 10 av. Ed. Belin 31055 Toulouse France damienne.bajon@isae.fr, \\ $\ddagger$ NXP-Semiconductors, Esplanade Anton Philips 14906, Colombelles Caen - France sidina.wane@ieee.org
}

\begin{abstract}
In this work we present the Correlation Transverse Wave Formulation (CTWF) method for direct computation of the auto- and cross correlation functions (ACFs and CCFs) of stationary stochastic electromagnetic fields. The transverse wave formulation, in performing a modal expansion of the electromagnetic fields in the homogeneous parts of the calculation domain and solving the near field continuity on both sides of the circuit surfaces, provides a direct derivation of the ACFs and CCFs without hypothesis on the structure of radiated fields.

Index Terms-Stochastic electromagnetic fields, electromagnetic interference, noisy electromagnetic fields, correlation transverse wave formulation.
\end{abstract}

\section{INTRODUCTION}

Radiated emissions in electromagnetic systems often originate from integrated circuits. An accurate near-field characterization of radiating electromagnetic structures is required along with modeling techniques which can efficiently handle noisy electromagnetic fields generated by these structures in order to facilitate an efficient computer aided development of electric and electronic systems [1]. In typical RF integratedcircuit (RFIC) structures, we encounter multilayer structures including inhomogeneous layer stacks with arbitrary doping profiles. The EM field in such structures can be efficiently modeled for deterministic field sources using the transverse wave formulation (TWF) [2]. Additionally to the field distributions on all the circuit surfaces, TWF provides an explicit expansion of the field at any level of the intermediate homogeneous parts and the surrounding facilitating explicit determination of cross correlation between sources.

For efficient EMI compliant design and optimization of circuits and systems, simulation methodologies based on the field autocorrelation and cross correlation spectral densities are required. Semi-analytic numerical methods based on Green's function formalism were already presented in [1], [3], [4]. Expansion of characterization of stationary noisy fields with Gaussian probability distribution to cyclostationary problems has been discussed in [5]. Characterization of noisy fields using field-field correlations opens new perspectives on signal integrity, EMI analysis, for low noise energy sensing and EMI source imaging [6]-[8]. Evolution of correlation information of stationary noisy propagating EM fields and aspects for their analysis by principal components are addressed in [9], [10].

In this work, we present a modeling approach based on TWF which handles the transformation of auto- and cross correlation information, which is necessary for the characterization of stochastic electromagnetic fields. In the following we review the TWF method. Then, we will present the correlation transverse wave formulation (CTWF) method, which expands the TWF method. Subsequently, we will demonstrate the method based on a numerical example.

\section{TWF - The Transverse Wave Formulation}

TWF may be referenced as an integral method in which EM fields are represented in Hilbert spaces. TWF solves integral equations on surfaces, the printed surfaces on which "the circuits" are defined, derived in terms of the tangential fields, i.e. the transverse field in reference to the outgoing and incoming normal of the circuit surface. Following [11], the homology between network theory and integral equation derivation is fully exploited and the Greens operator between conjugate electromagnetic fields is associated to homogeneous spaces on both sides or between circuit surfaces. An extension of this formalism has been given in the transverse wave formulation [2], [12]-[14]. More specifically, the TWF handles an additive and subtractive combination of tangential electric and magnetic fields, i.e. transverse waves, and in this sense follows the Transmission Line Matrix (TLM) method which may be considered as its differential counterpart. In the context of integral operators, the choice to formulate the problem in terms of waves has two major consequences: involved Green's operators are bounded integral operators which prevent any singularity issues and, on the other hand, continuity conditions across a surface have the general form of an optic-like transfer condition. These properties are proven to be crucial when establishing the computational scheme of the boundary value problem defined by the circuit pattern [2], [12], [13].

As in any other integral formulations, in the TWF approach only the surfaces defined by the circuit are discretized on both faces, while the EM fields, once solved for, are explicitly available at any point of the calculation domain through postprocessing of the Green's function. This property allows to contain the computational complexity of problems and is decisive in calculating auto and cross correlations or coherences between any points of the total volume. Moreover, the Correlation Green's Function (CGF) is obtained without any hypothesis on field variations, according to the distance to electric or magnetic current sources.

At the circuit surfaces, the EM fields are given in terms of an expansion in the eigenfunctions of the Green's operator used to set the integral equation. Let us consider the modal expansion 
for a wave-guide as depicted in Fig. 1a). The field inside this waveguide can be decomposed into its modal eigensolutions. These modal solutions represent the spectral expansion of the Green's operator associated with the homogeneous domains. They provide the "analytical" expression for the expansion of the fields in any parts of the calculation volume around and in between the circuit surfaces, where the tangential field components are explicitly discretized and solved.

We introduce the electric and magnetic structure forms $e_{k}(u, v)$ and $h_{k}(u, v)$, in order to express the transverse field forms as products of these structure forms, with complex amplitudes depending on the $z$-coordinate. For the exterior differential form formalism applied in the following we refer to [14]. The index $k$ provides a compact notation for the double index $m n$ and the transverse $\mathrm{TE}_{m n}$ and $\mathrm{TM}_{m n}$ modes in the case where the cross section of the cylindrical structure in Fig. 1a) is homogeneous. The structure forms $e_{k}(u, v)$ and $h_{k}(u, v)$ form a normalized biorthogonal basis in the more general case where the cross section of the cylindrical structure in Fig. 1a) is inhomogeneous

$$
\left\langle e_{k} \mid h_{l}\right\rangle_{A}=-\left\langle h_{k} \mid e_{l}\right\rangle_{A}=\delta_{k l} .
$$

We can represent the complete electric and magnetic fields by Hilbert space vectors as

$$
\begin{aligned}
\left|\underline{\mathcal{E}}_{t}(z)\right\rangle & =\sum_{k}\left(\underline{V}_{k}^{(+)} e^{-\gamma_{k} z}+\underline{V}_{k}^{(-)} e^{\gamma_{k} z}\right)\left|e_{k}\right\rangle, \\
\left|\underline{\mathcal{H}}_{t}(z)\right\rangle & =\sum_{k} \frac{1}{Z_{W, k}}\left(\underline{V}_{k}^{(+)} e^{-\gamma_{k} z}-\underline{V}_{k}^{(-)} e^{\gamma_{k} z}\right)\left|h_{k}\right\rangle .
\end{aligned}
$$

Hence, the electric and magnetic field forms can be obtained from the above Hilbert space vectors as

$$
\begin{aligned}
\underline{\mathcal{E}}_{t}(\boldsymbol{x}) & =\sum_{k} e_{k}(u, v)\left\langle h_{k} \mid \underline{\mathcal{E}}_{t}\right\rangle, \\
\underline{\mathcal{H}}_{t}(\boldsymbol{x}) & =\sum_{k} \frac{1}{Z_{W, k}} h_{k}(u, v)\left\langle e_{k} \mid \underline{\mathcal{H}}_{t}\right\rangle,
\end{aligned}
$$

where $\boldsymbol{x}$ is the vector containing the space coordinates. With this we introduce the wave amplitude vectors $|\underline{\tilde{\mathcal{A}}}\rangle$ and $|\underline{\tilde{\mathcal{B}}}\rangle$ as

$$
\begin{aligned}
|\underline{\tilde{\mathcal{A}}}\rangle & =\frac{1}{2}\left[\left|\underline{\mathcal{E}}_{t}\right\rangle+\boldsymbol{Z}_{W}\left|\underline{\mathcal{H}}_{t}\right\rangle\right], \\
|\underline{\tilde{\mathcal{B}}}\rangle & =\frac{1}{2}\left[\left|\underline{\mathcal{E}}_{t}\right\rangle-\boldsymbol{Z}_{W}\left|\underline{\mathcal{H}}_{t}\right\rangle\right],
\end{aligned}
$$

where we use the wave impedance operator defined as

$$
\boldsymbol{Z}_{W}=\sum_{k=1}^{\infty} Z_{W, k}\left(\left|h_{k}\right\rangle\left\langle e_{k}|-| e_{k}\right\rangle\left\langle h_{k}\right|\right) .
$$

The amplitudes of the transverse electric and magnetic fields are computed from the wave amplitudes via

$$
\begin{aligned}
\left|\underline{\mathcal{E}}_{t}\right\rangle & =|\tilde{\mathcal{\mathcal { A }}}\rangle+|\underline{\tilde{\mathcal{B}}}\rangle, \\
\left|\underline{\mathcal{H}}_{t}\right\rangle & =Z_{W}^{-1}(|\underline{\tilde{\mathcal{A}}}\rangle-|\underline{\tilde{\mathcal{B}}}\rangle) .
\end{aligned}
$$
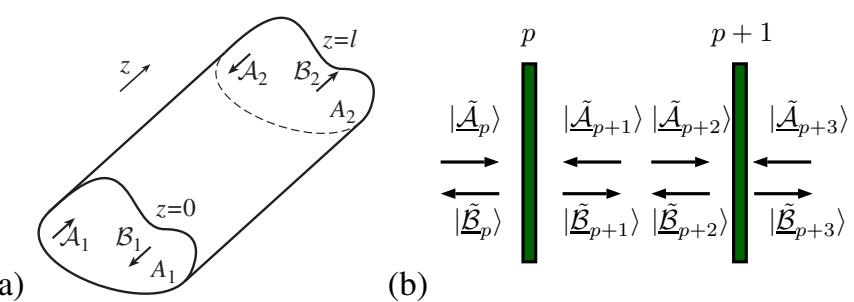

(b)

Fig. 1: Wave amplitudes in the waveguide segment (a) and multilayer structure with interfaces $p \ldots p+1$ and incident and reflected waves (b).

We introduce the operator $\boldsymbol{\Gamma}(z)$ and its inverse as

$$
\begin{aligned}
\boldsymbol{\Gamma}(z) & =\sum_{n=1}^{\infty} e^{-\gamma_{k} z}\left(\left|h_{n}\right\rangle\left\langle e_{n}|-| e_{n}\right\rangle\left\langle h_{n}\right|\right), \\
\boldsymbol{\Gamma}^{-1}(z) & =\sum_{n=1}^{\infty} e^{\gamma_{k} z}\left(\left|h_{n}\right\rangle\left\langle e_{n}|-| e_{n}\right\rangle\left\langle h_{n}\right|\right) .
\end{aligned}
$$

The direction of the wave amplitude vectors at the ports of the waveguide segment are assigned as shown in Figure 1a). For homogeneous layers between the interfaces $p$ and $p+1$, see Fig. 1b), incident and reflected waves are related through a reflection operator in a two port form [2], [14]

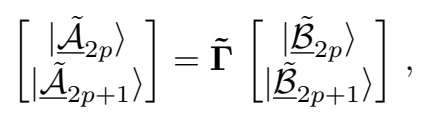

where the reflection operator $\tilde{\boldsymbol{\Gamma}}$ is derived from the Green's admittance operator $\tilde{\boldsymbol{Y}}$ of the homogeneous media and has the form

$$
\tilde{\boldsymbol{\Gamma}}=\left[\begin{array}{ll}
\boldsymbol{\Gamma}_{11}\left(z_{1}-z_{2}\right) & \boldsymbol{\Gamma}_{12}\left(z_{1}-z_{2}\right) \\
\boldsymbol{\Gamma}_{21}\left(z_{1}-z_{2}\right) & \boldsymbol{\Gamma}_{22}\left(z_{1}-z_{2}\right)
\end{array}\right]
$$

On a layer $p$ (Fig. 1), which represents an inhomogeneous layer of small thickness or printed surface of the circuit, incident waves are scattered

$$
\left[\begin{array}{c}
\left|\tilde{\mathcal{B}}_{2 p}\right\rangle \\
\left|\underline{\tilde{\mathcal{B}}}_{2 p+1}\right\rangle
\end{array}\right]=\tilde{\boldsymbol{S}}\left[\begin{array}{c}
\left|\tilde{\mathcal{\mathcal { A }}}_{2 p}\right\rangle \\
\left|\underline{\tilde{\mathcal{\mathcal { A }}}}_{2 p+1}\right\rangle
\end{array}\right],
$$

where $\tilde{\boldsymbol{S}}$ is the scattering operator built from the continuuity conditions of the tangential fields [2], [14]. Collecting all the incoming and outgoing waves in (8) and (10) of all the interfaces in the structure, the Hilbert space vectors of incident and reflected waves are built and the problem is settled in the form of coupled equations

$$
\begin{aligned}
|\underline{\tilde{\mathcal{A}}}\rangle & =\tilde{\boldsymbol{\Gamma}}|\underline{\tilde{\mathcal{B}}}\rangle, \\
|\underline{\tilde{\mathcal{B}}}\rangle & =\tilde{\boldsymbol{S}}|\underline{\tilde{\mathcal{A}}}\rangle+\left|\underline{\tilde{\mathcal{B}}}_{0}\right\rangle,
\end{aligned}
$$

where $\left|\tilde{\mathcal{B}}_{0}\right\rangle$ describes all wave source terms. The resolution scheme solves iteratively conditions (11) and (12) providing the implicit inversion of the operator $M$ associated to the integral equation of the boundary value problem

$$
|\underline{\tilde{\mathcal{B}}}\rangle=(1-\tilde{\boldsymbol{S}} \tilde{\boldsymbol{\Gamma}})^{-1}\left|\underline{\tilde{\mathcal{B}}}_{0}\right\rangle \equiv M\left|\tilde{\tilde{\mathcal{B}}}_{0}\right\rangle .
$$


In this description of the layered electromagnetic structure, the generalized voltages and currents and the wave amplitudes are summarized in Hilbert space vectors describing the complete transverse field distribution [2], [12], [15]. Using this formalism we can analyze multilayer structures, where homogeneous layers of finite extension in $z$-direction alternate with thin structured layers. The structured layers are described by the surface admittance matrices $Y_{i j}(u, v)$ translated in scattering operators as presented in (10). From this formulation, we can describe multilayer structures consisting of thin layers, with structured metalization, resistive layers, and impressed voltage and current sources and thick homogeneous material. For a detailed description of the method the reader is referred to [2].

\section{CTWF - The CoRrelation TWF}

We consider stationary noisy fields with Gaussian probability distribution. A Gaussian process can be fully described by its mean value and its second-order moments. Hence, in order to describe stationary stochastic EM fields, we introduce the correlation dyadics

$$
\begin{aligned}
& C^{B_{0}}=\overline{\left|\tilde{\mathcal{B}}_{0}\right\rangle\left\langle\underline{\mathcal{B}}_{0}\right|}, \\
& C^{B}=|\underline{\tilde{\mathcal{B}}}\rangle\langle\underline{\tilde{\mathcal{B}}}|,
\end{aligned}
$$

where the overline denotes the ensemble average. The diagonal elements of (14), i.e. the autocorrelation terms, represent the spectral power. With the structure forms introduced above, the spectral energy density can be obtained at defined ports of the structure. From (13) and (14) we obtain (see also [1], [16]) the bilinear equation

$$
\boldsymbol{C}^{B}=\boldsymbol{M} \boldsymbol{C}^{B_{0}} \boldsymbol{M}^{\dagger} .
$$

This relation governs the transformation of the correlation dyadics describing the stochastic EM field.

\section{NumericAl ExAmple AND APPLICATION}

A numerical example is presented with an arrangement of two sources. Five observation points $S_{1} \ldots S_{5}$ are arranged in a linear array with a spacing of $5 \mathrm{~mm}$. The two sources $S_{6}$ and $S_{7}$ are placed in a distance of $15 \mathrm{~mm}$ from this linear array as depicted in Fig. 2. Correlation data are computed for the five observation points receiving EMI from two sources with chosen degree of correlation. The excitation signal is given by rectangular function in the frequency domain with $10 \mathrm{GHz}$ bandwidth. Upon determining $M$ via the transverse wave formulation, the field correlation at the observation points $S_{1} \ldots S_{5}$ is determined applying (15). The real part of the frequency domain auto correlation function for the observation points is plotted in Fig. 3 for two type of source correlations. The upper plot shows a correlated in-phase excitation of the sources $S_{6}$ and $S_{7}$ yielding a maximum in spectral energy density for the observation point $S_{3}$. The second plot shows a correlated anti-phase excitation resulting, as anticipated, in a minimum for the spectral energy density for observation point $S_{3}$. With this formulation, arbitrary degrees of correlations

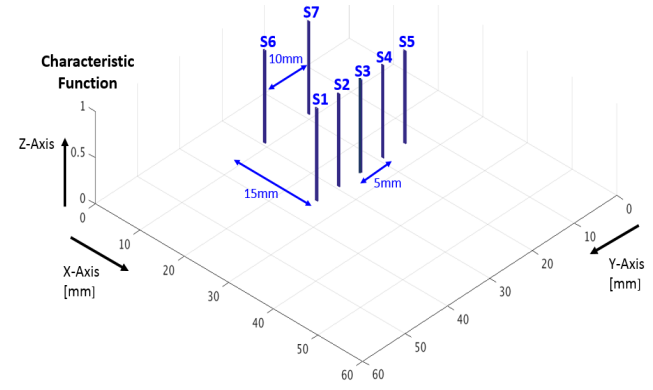

Fig. 2: Arrangement of source and observation points.
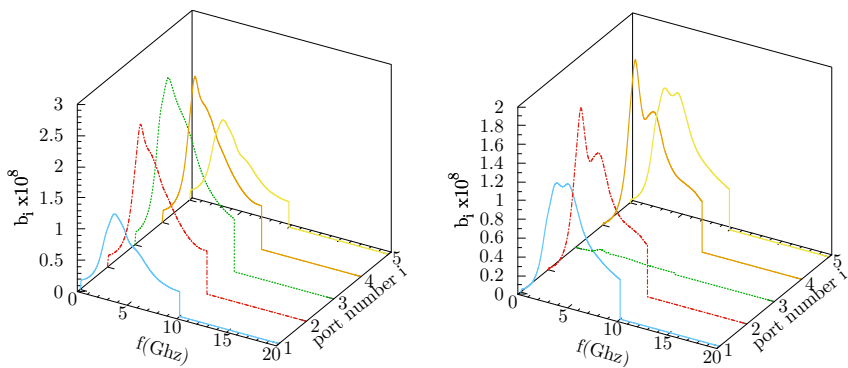

Fig. 3: In-phase (left) and anti-phase excitation (right).

between the source terms can be considered, also determined from measurement, and the resulting energy densities can be computed at the observation points.

Two practical applications are investigated namely near-field propagation of noisy Gaussian sources and radiated emissions from a CPW transmission line structure. The Gaussian sources are modeled in TWF using modal representation expanded in pixel-like basis functions with a resolution $\delta x$ as illustrated in Fig. 4(a). The reference noisy Gaussian sources are extracted from measurement using coupled horn antenna elements (Fig. 4(a)) and imported in TWF based on modepixel transform. Fig. 4(b) presents the comparison between the measurement of the normalized transverse field and the model generated for incorporation in TWF resolution process. The CPW line is modeled using delta-gap excitation sources. In Figs. 4(a) and (b), correlation between modeling results and measurement for the variation of the transverse magnetic field at $3 \mathrm{GHz}$ as function of the height and as function of the $y$-coordinate is presented showing good agreement. Both applications are suitable for using auto-correlation and crosscorrelation functions as means for analyzing effects of nearfield propagation accounting for diffraction and dislocation effects [17].

\section{CONClusion}

In this work, we introduced and applied the Correlation Transverse Wave Formulation (CTWF) method for direct computation of the auto and cross correlation functions of stationary stochastic electromagnetic fields.

The TWF method is an efficient tool for modeling inhomogeneous layer stacks. It works with spatial and spectral expansions of the field problem and provides a natural framework 


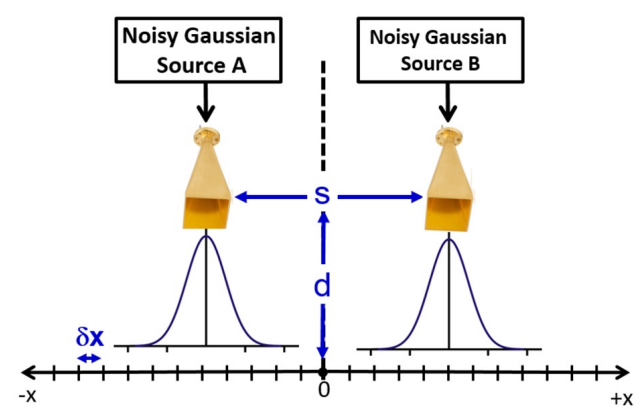

(a)

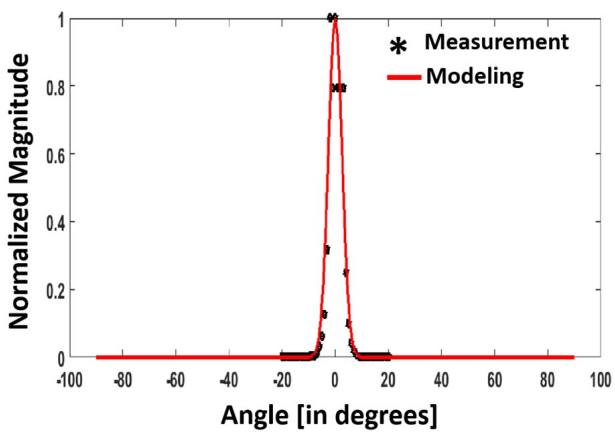

(b)

Fig. 4: Experimental setup using two noisy Gaussian sources $\mathrm{A}$ and $\mathrm{B}$ (a). Comparison between measurement of the normalized transverse field and the generated noisy model incorporation in TWF resolution process (b).

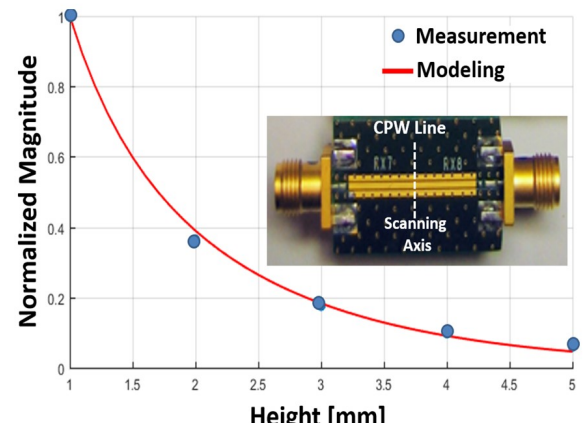

(a)

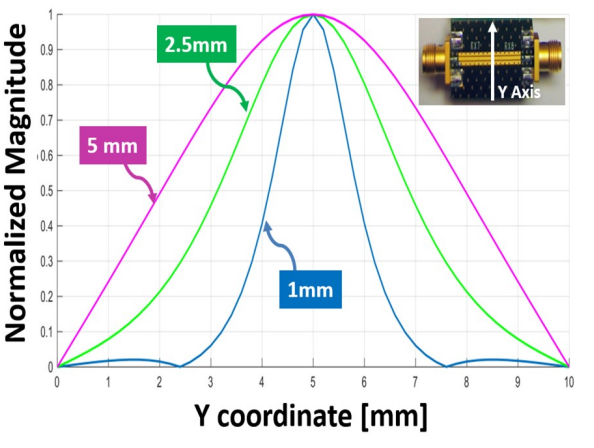

(b)

Fig. 5: Measured and simulated variations of normalized magnetic field at the center of the CPW line at $3 \mathrm{GHz}$ as function of height (a) and $y$-coordinate (b). for handling multi-resolution and multi-scale problems.

Correlation analysis provides a basis for accurate modeling of noisy electromagnetic fields and we have demonstrated its direct incorporation to the transverse wave approach. The CTWF method provides an efficient technique to compute stochastic electromagnetic fields in layer stacks.

\section{ACKNOWLEDGEMENT}

This work was supported by the European Union's Horizon 2020 research and innovation programme under grant no. 664828 (NEMF21).

\section{REFERENCES}

[1] J. A. Russer and P. Russer, "Modeling of noisy EM field propagation using correlation information," IEEE Transactions on Microwave Theory and Techniques, vol. 63, no. 1, pp. 76 - 89, Jan. 2015.

[2] S. Wane, D. Bajon, H. Baudrand, and P. Gamand, "A new full-wave hybrid differential-integral approach for the investigation of multilayer structures including nonuniformly doped diffusions," IEEE Trans. Microwave Theory Techn., vol. 53, no. 1, pp. 200 - 214, Jan. 2005.

[3] J. A. Russer and P. Russer, "Stochastic electromagnetic fields," in German Microwave Conference (GeMIC), Mar. 2011, pp. 1-4.

[4] G. Gradoni, S. C. Creagh, G. Tanner, C. Smartt, and D. W. P. Thomas, "A phase-space approach for propagating field-field correlation functions," New Journal of Physics, vol. 17, no. 9, p. 093027, 2015.

[5] J. A. Russer, P. Russer, M. Konovalyuk, A. Gorbunova, A. Baev, and Y. Kuznetsov, "Near-field propagation of cyclostationary stochastic electromagnetic fields," in Int. Conf. on Electromagnetics in Adv. Applications (ICEAA), 2015, Torino, Italy, Sep. 7-11 2015, pp. 1456-1459.

[6] S. Wane, O. Doussin, D. Bajon, J. Russer, and P. Russer, "Stochastic approach for power integrity, signal integrity and EMC/EMI analysis of moving objects," in Int. Conf. on Electromagnetics in Adv. Applications (ICEAA), 2015, Torino, Italy, Sep 7-11 2015, pp. 1554-1557.

[7] S. Wane, D. Bajon, J. Russer, P. Russer, and J. M. Moschetta, "Concept of twin antenna-probe using stochastic field-field $\mathrm{x}$-correlation for energy sensing and low-noise blind deconvolution," in 2016 IEEE Conference on Antenna Measurements Applications (CAMA), Oct 2016, pp. 1-4.

[8] J. A. Russer and P. Russer, "Imaging of sources of radiated electromagnetic interference," Frequenz, vol. 65, pp. 261-265, Sep. 2011.

[9] J. A. Russer, G. Gradoni, G. Tanner, S. C. Creagh, D. Thomas, C. Smartt, and P. Russer, "Evolution of transverse correlation in stochastic electromagnetic fields," in Proceeding of: IEEE International Microwave Symposium, IMS, Phoenix, Arizona, USA, May 17-22 2015.

[10] M. Haider and J. A. Russer, "Principal component analysis for efficient characterization of stochastic electromagnetic fields," Int. J. of Num. Modelling, Electronic Networks, Devices and Fields, accepted for publication 2017.

[11] H. Baudrand and D. Bajon, "Equivalent circuit representation for integral formulation of electromagnetic problems," Int. J. of Num. Modelling, Electronic Networks, Devices and Fields, vol. 15, pp. 23-57, 2002.

[12] S. Wane, D. Bajon, and H. Baudrand, "Fullwave analysis of stacked structures including inhomogeneous antennas," in New Trends and Concepts in Microwave Theory and Technics, H. Baudrand, Ed. Trivandrum, India: Research Signpost, 2003, pp. 131-166.

[13] D. Bajon, S. Wane, and H. Baudrand, "Multi-scale investigation of global coupling between blocks and sub-blocks including lumped components in RF integrated circuits," Proc. 34th European Microwave Conference, Amsterdam, vol. 2, pp. 873 - 875, Oct. 2004.

[14] P. Russer, Electromagnetics, Microwave Circuit and Antenna Design for Communications Engineering, 2nd ed. Boston: Artech House, 2006.

[15] H. Baudrand, Introduction au Calcul des Elements de Circuits Passifs en Hyperfreequences. Toulouse: Cépaduès-Éditions, 2001.

[16] H. Hillbrand and P. Russer, "An efficient method for computer aided noise analysis of linear amplifier networks," IEEE Trans. Circuits and Systems, vol. 23, no. 4, pp. 235-238, Apr. 1976.

[17] I. D. Maleev and J. G. A. Swartzlander, "Propagation of spatial correlation vortices," J. Opt. Soc. Am. B, vol. 25, no. 6, pp. 915-922, Jun 2008. 